\title{
Detection of Cardiac Arrhythmias From Varied Length Multichannel Electrocardiogram Recordings Using Deep Convolutional Neural Networks
}

\author{
Marwen Sallem $^{1}$, Amina Ghrissi ${ }^{2}$, Adnen Saadaoui $^{3}$ and Vicente Zarzoso ${ }^{2}$ \\ ${ }^{1}$ National Institute of Applied Sciences and Technology, MMA Laboratory, Tunisia \\ ${ }^{2}$ Université Côte d'Azur, CNRS, I3S Laboratory, Sophia Antipolis, France \\ ${ }^{3}$ Vneuron,Tunisia
}

\begin{abstract}
Automatic identification of different arrhythmias helps cardiologists better diagnose patients with cardiovascular diseases. Deep learning algorithms are used for the classification of multichannel ECG signals into different heart rhythms. The study dataset includes a cohort of 43101 12-lead ECG recordings with various lengths. Two options are tested to standardize the recordings length: zero padding and signal repetition. Downsampling the recordings to $100 \mathrm{~Hz}$ allow handling the problem of different sampling frequencies of data coming from different sources. We design a deep one-dimensional convolutional neural network (CNN) called VGG-ECG, a 13-layer fully CNN for multilabel classification. Our team is called MIndS and our approach achieved a challenge validation score of 0.368 , and full test score of -0.128 , placing us 38 out of 41 in the official ranking.
\end{abstract}

\section{Introduction}

Cardiovascular diseases represent a major cause of death worldwide as they are responsible for about one million deaths annually in the United States alone [1]. Hence, contributing to the automatic identification of different arrhythmias with deep learning (DL) tools [2] would help cardiologists better diagnose patients with cardiovascular diseases. For this reason, the current work aims to classify multichannel ECG signals into 27 heart rhythms using DL as part of Pyhsionet Challenge 2020 [3] [4]. The study dataset provided by the challenge includes a cohort of 43101 12-lead ECG recordings of different heart rhythms. The main issues encountered in the classification task consist in the varied lengths of multichannel ECG recordings added to the difference in characteristics of samples coming from different data sources.

We design a deep one dimensional (1D) convolutional neural network (CNN) inspired by VGG16 architecture (developed by Visual Geometry Group) [5] [6] and reg- ularized with dropout [7]. Data preprocessing and preparation is also performed. Long recordings are truncated and only the first 3000 time samples are kept to alleviate the training load. Two options are tested to standardize the recording lengths: zero padding and signal repetition. The designed solution is expected to help cardiologists distinguish 27 scored cardiac rhythms out of 111 .

\section{Methods}

\subsection{Classification Metrics}

A variety of metrics is used to evaluate the classification performance. In the unofficial phase of the challenge, metrics used include: F2-score, G2-score and geometric mean (GM). The metrics formulas combine precision and recall [4]. In the official phase, one single score $(S)$ is computed. $S$ generalizes the traditional accuracy metric by giving full credit to correct diagnoses and penalizing wrong diagnosis, with respect to similarities between arrhythmias:

$$
S=\sum_{i}^{j} w_{i j} a_{i j},
$$

where $a_{i j}$ is an element of the confusion matrix equal to the number of samples classified as belonging to category $c_{i}$ but that truly belong to $c_{j}$. Moreover, different weights $W=\left[w_{i j}\right]$ are assigned to a couple of classes $\left(c_{i}, c_{j}\right)$ based on their similarity levels. The higher the values of the scores, the better our model [8].

\subsection{VGG-ECG}

VGG-ECG is a 13-layer fully CNN composed of blocks of 2 and 3 successive convolutional (Conv) layers alternated by average pooling layers. 1D Conv layers have a receptive field of dimensions $1 \times 3$ to scan information along the temporal dimension of the leads. They are denoted Conv3 as shown in the Fig. 1. This architecture 


\begin{tabular}{|c|}
\hline Conv3 x 64 \\
\hline Conv3 x 64 \\
\hline Pooling \\
\hline Conv3 x 128 \\
\hline Conv3 x 128 \\
\hline Pooling \\
\hline Conv3 x 256 \\
\hline Conv3 x 256 \\
\hline Conv3 x 256 \\
\hline Pooling \\
\hline Conv3 x 256 \\
\hline Conv3 x 256 \\
\hline Conv3 x 256 \\
\hline Pooling \\
\hline Conv3 x 256 \\
\hline Conv3 x 256 \\
\hline Conv3 x 256 \\
\hline Pooling \\
\hline
\end{tabular}

Figure 1. VGG-ECG architecture [5].

proved efficient in a similar multichannel ECG classification task [5]. Dropout with a drop probability equal to 0.3 is used to regularize the model and avoid overfitting [7]. ADAM optimizer with learning rate equal to $10^{-3}[9]$ is used for training. Binary cross-entropy loss is also used to allow multilabel classification of recordings in more than one class $\left(c_{i}\right)$, This cost function proved more efficient than mean squared error that gives poor results. Random seeds are fixed in order to make experiments reproducible when training on GPUs.

Implementation: DL learning experiments are conducted with the use of Python 3.7 programming language and Tensorflow 2.3 library within Google Cloud Platform (GCP), running on n1-standard-4 (4 vCPUs, 15 GB memory) machine and NVIDIA Tesla T4 Virtual Workstation GPU [11]. Wandb [12] ML experiment tracking tool is used to assess the training performance and visualize statistics about the model.

\subsection{Data Preparation}

The public dataset afforded by the challenge committee for training and validation is composed of 43101 12-lead ECG recordings coming from four different data sources. Recordings have different lengths varying from $10 \mathrm{~s}$ to $30 \mathrm{~min}$. The sampling frequencies $\left(F_{s}\right)$ are also different and depend on the data sources as described in [4].

In order to train the model, we consider the majority of the signals coming from the four data sources. We select the signals belonging to at least one of the 27 scored classes. Hence, we passed from 43101 to 37794 entry signals. The training is performed on $3 / 4$ of the dataset, while the remaining samples are used for validation.

Regarding the difference of the recording lengths that vary from $10 \mathrm{~s}$ to $30 \mathrm{~min}$, we propose to consider only the first 3000 temporal samples and ignore the rest. We expect the first part of each recording to contain sufficient information about the heart rhythm. Signals that are shorter than 3000 samples are zero padded. Truncating the first 3000 samples after downsampling allows us to alleviate considerably the computational load of training and validation process.

In order to handle the problem of different sampling frequencies, we aim to standardize $F_{s}$ by downsampling all recordings to a standard frequency $\left(F_{d}\right)$. We benchmark the classification performance on the test set with respect to three values of $F_{d}$ as shown in Tab. 1. $F_{d}=100 \mathrm{~Hz}$ provides better performance than 50 and $150 \mathrm{~Hz}$.

\begin{tabular}{|c|c|c|c|}
\hline$F_{d}(\mathrm{~Hz})$ & 50 & 100 & 150 \\
\hline$S$ & 0.25 & 0.47 & 0.44 \\
\hline
\end{tabular}

Table 1. Effect of $F_{d}$ on test classification scores.

\section{Experiments and Results}

Initial experiments are conducted on the preliminary dataset afforded for the unofficial submission phase of Physionet challenge. Then final results are conducted on the full dataset.

\subsection{Preliminary Results}

In order to handle class imbalance, class weights of the neural networks nodes are configured to ensure balance. Data preprocessing includes zero padding, considering only the first 18000 samples of the padded recordings. The training scores F2-score, G2-score and geometric mean (GM) after 5-fold cross validation are respectively $0.77,0.58$ and 0.67 with zero padding and $0.74,0.54$ and 0.63 with signal repetition. The scores' standard deviations are below 0.02 . By analyzing the confusion matrix, we notice that the ST-segment elevation (STE) class has poor sensitivity (0.43). This can be explained by the low number of samples (220) compared to atrial fibrillation, for instance, that has 1221 samples and high sensitivity (0.93). Results on the test set are F2-score $=0.77, \mathrm{G} 2$-score $=0.55$ and $\mathrm{GM}=0.65$. 


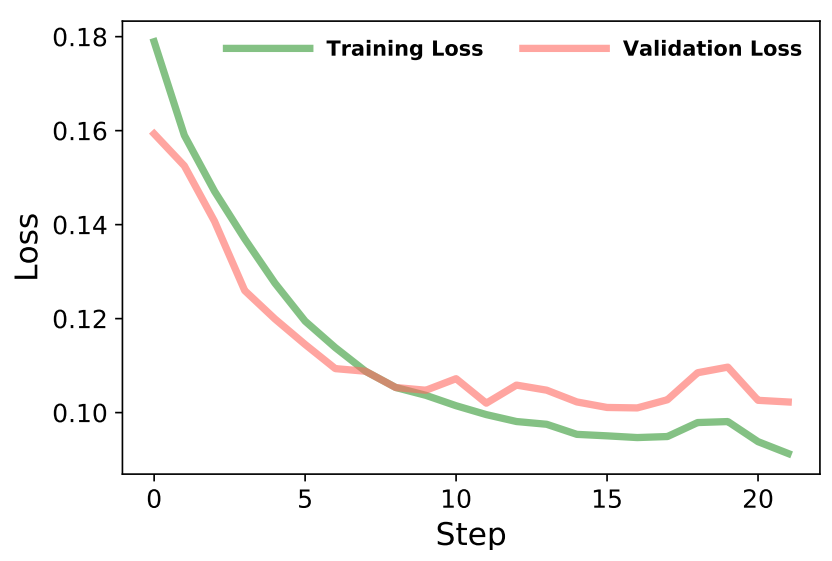

Figure 2. Training and Validation curve.

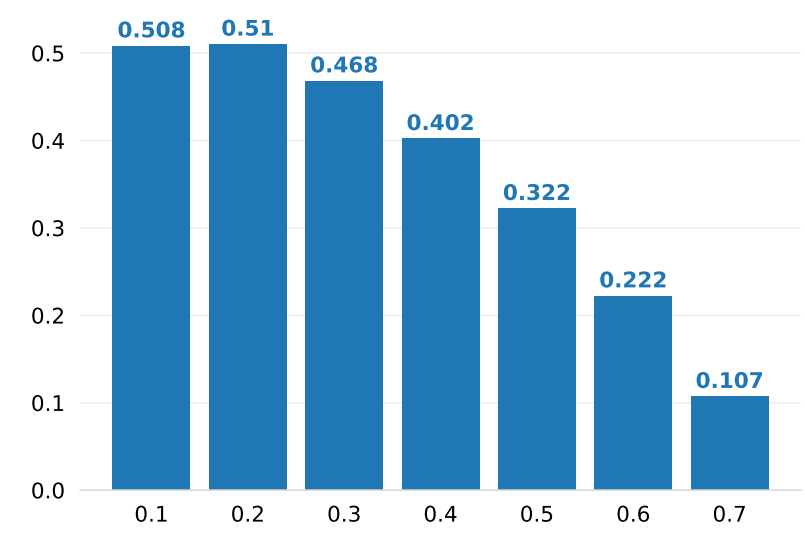

Figure 3. The threshold effect.

\subsection{Final Results}

The training consists in several steps and each step is a full pass over the entire dataset. In order to avoid overfitting, training is stopped when the value of validation loss does not improve significantly for more than five steps. Then the trained model reaching the best performance is selected. The retained model achieved a validation accuracy of 0.54 and validation loss of 0.10 after $2 \mathrm{~h} 30 \mathrm{~min}$ of training through 21 step as shown in Fig. 2 .

The threshold has a direct impact on the computed score $S$. Several values of thresholds are evaluated on the test set. Fig. 3 shows that for a high threshold value, the score gets lower. Indeed, the score metric penalizes more the false negatives compared to the false positives. We select a threshold value equal to 0.2 giving the best score in the final model.

Then, the model is submitted to the challenge committee

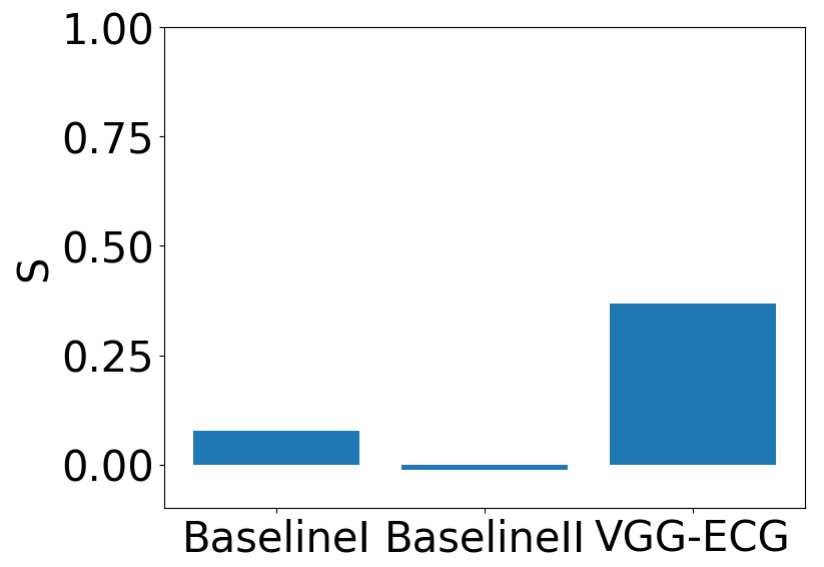

Figure 4. Validation classification scores.

to be assessed on hidden validation and test sets. The results are shown in Fig. 4. VGG-ECG reaches $S=0.37$ compared to baseline models (Baseline I and II), proposed by the challenge, which scores' values do not exceed 0.1 . This result can be explained by the ability of CNNs to capture complex hidden features from data compared to baseline models based on handcrafted features and logistic regression [10].

We are the MIndS team and we achieved a full test score of -0.128 which places us 38 out of 41 in the official ranking.

\section{Conclusions and perspectives}

The classification of multichannel ECG recordings using DL can help cardiologists automatically detect different arrhythmia types added to sinus rhythm. In order to handle the varied length recordings and the imbalanced dataset issues, we benchmark several solutions. Two options are tested to standardize the recordings length: zero padding and signal repetition. Moreover, all signals are downsampled to $100 \mathrm{~Hz}$ in order to standardize the different sampling frequencies. Several settings are also assessed such as adding residual layers and customizing classification thresholds for each class but these approaches did not enhance the performance. Moreover, preprocessing methods like low-pass filtering and standard normalisation does not help achieving better scores. The multilabel classification performed considers only the 27 scored classes, as stated by the challenge guidelines, and ignores the remaining classes. By benchmarking the classification performance of several architectures, we opt for a deep 1D CNN model inspired by VGG16 for multilabel classification with binary cross entropy loss. However, the score on the hidden test set is equal to -0.128 .

Further work will investigate data augmentation [13] [14] technique to handle the issue of imbalanced dataset ratio 
between different arrhythmias. Also, we will add LSTM and attention layers to the CNN model as they are expected to learn automatically salient dependencies from ECG time series [5] [15].

\section{Acknowledgments}

The work of Amina Ghrissi is partly funded by the French government PIA program, IDEX UCA ${ }^{\text {JEDI }}$ project (ANR-15-IDEX-0001).

\section{References}

[1] C. T. January, et al. "2014 AHA/ACC/HRS guideline for the management of patients with atrial fibrillation: a report of the American College of Cardiology. American Heart Association Task Force on Practice Guidelines and the Heart Rhythm Society," Journal of the American College of Cardiology, 64(21), 2014.

[2] Goodfellow I, Bengio Y, Courville A. Deep Learning, MIT Press, 2016. www.deeplearningbook.org.

[3] Goldberger A, et al. PhysioBank, PhysioToolkit, and PhysioNet: Components of a new research resource for complex physiologic signals. Circulation [Online]. 101(23), pp. 215-220.

[4] Perez A. EA, et al. "Classification of 12-lead ECGs: the PhysioNet/Computing in Cardiology Challenge 2020". Physiol. Meas, 2020.

[5] Qihang Y, et al. "Multi-class arrhythmia detection from 12-lead varied-length ECG using Attention-based TimeIncremental Convolutional Neural Network." Information Fusion, 53(2020): pp. 174-182.

[6] Simonyan K, Zisserman A. "Very deep convolutional networks for large-scale image recognition," In Proc. International Conference on Learning Representations, San Diego, CA, 2015.

[7] Srivastava N, et al. "Dropout: a simple way to prevent neural networks from overfitting," The Journal of Machine Learning Research, 15(1), pp. 1929-1958, 2014.

[8] Sokolova M, Lapalme G. "A systematic analysis of performance measures for classification tasks." Information Processing and Management, 45(4), p. 427-437, 2009.

[9] Changyou C, et al. "Bridging the gap between stochastic gradient MCMC and stochastic optimization." Artificial Intelligence and Statistics, 2016.

[10] Dreiseitl S, Lucila OM. "Logistic regression and artificial neural network classification models," Journal of Biomedical Informatics, 35(5-6), pp. 352-359, 2002.

[11] E. Lindholm, et al. "NVIDIA Tesla: A Unified Graphics and Computing Architecture." in IEEE Micro, 28( 2), pp. 39-55, 2008.

[12] L. Biewald, Experiment Tracking with Weights and Biases. Software available from wandb.com 2020.

[13] Shorten C, Khoshgoftaar TM. "A survey on image data augmentation for deep learning." Journal of Big Data, 6(1), p. 60, 2019.
[14] Agnieszka A, Grochowski M, "Data augmentation for improving deep learning in image classification problem." In Proc. International Interdisciplinary PhD Workshop, IEEE, 2018.

[15] Hannun AY, et al. "Cardiologist-level arrhythmia detection and classification in ambulatory electrocardiograms using a deep neural network," Nature Medicine, 25, pp. 65-69, 2019.

Address for correspondence:

Amina Ghrissi

Université Côte d'Azur, CNRS, I3S Laboratory

Les Algorithmes, Euclide B, 06103, Sophia Antipolis, France amina.ghrissi@univ-cotedazur.fr 